\title{
Surgical stabilization of flail chest injuries with MatrixRIB implants: A prospective observational study
}

\author{
Michael Bottlang a,*, William B. Long ${ }^{a}$, Daniel Phelan ${ }^{\mathrm{a}}$, Drew Fielder ${ }^{\mathrm{b}}$, Steven M. Madey ${ }^{\mathrm{a}}$ \\ ${ }^{a}$ Legacy Research Institute, 1225 NE 2nd Ave, Portland, OR 97232, United States \\ b 1400 N. IH 35, Ste 300, Austin, TX 78701, United States
}

\section{A R T I C L E I N F O}

\section{Article history:}

Accepted 31 July 2012

\section{Keywords:}

Flail chest

Rib fracture

Plate

Splint

Osteosynthesis

Prospective study

Blunt chest trauma

Osteosynthesis

\begin{abstract}
A B S T R A C T
Background: Surgical stabilization of flail chest injury with generic osteosynthesis implants remains challenging. A novel implant system comprising anatomic rib plates and intramedullary splints may improve surgical stabilization of flail chest injuries. This observational study evaluated our early clinical experience with this novel implant system to document if it can simplify the surgical procedure while providing reliable stabilization.

Methods: Twenty consecutive patients that underwent stabilization of flail chest injury with anatomic plates and intramedullary splints were prospectively enrolled at two Level I trauma centres. Data collection included patient demographics, injury characterization, surgical procedure details and postoperative recovery. Follow-up was performed at three and six months to assess pulmonary function, durability of implants and fixation and patient health.

Results: Patients had an Injury Severity Score of $28 \pm 10$, a chest Abbreviated Injury Score of $4.2 \pm 0.4$ and $8.5 \pm 2.9$ fractured ribs. Surgical stabilization was achieved on average with five plates and one splint. Intraoperative contouring was required in $14 \%$ of plates. Post-operative duration of ventilation was $6.4 \pm 8.6$ days. Total hospitalization was $15 \pm 10$ days. At three months, patients had regained $84 \%$ of their expected forced vital capacity (\%FVC). At six months, 7 of 15 patients that completed follow-up had returned to work. There was no mortality. Among the 91 rib plates, 15 splints and 605 screws in this study there was no hardware failure and no loss of initial fixation. There was one incidence of wound infection. Implants were removed in one patient after fractures had healed.

Conclusions: Anatomic plates eliminated the need for extensive intraoperative plate contouring. Intramedullary rib splints provided a less-invasive fixation alternative for single, non-comminuted fractures. These early clinical results indicate that the novel implant system provides reliable fixation and accommodates the wide range of fractures encountered in flail chest injury.
\end{abstract}

(c) 2012 Elsevier Ltd. All rights reserved.

\section{Introduction}

Flail chest injury is diagnosed by paradoxical motion of an incompetent chest wall segment, comprised of three or more consecutive ribs that are fractured in at least two places. Flail chest is present in $6-15 \%$ of patients that have sustained blunt chest wall trauma. ${ }^{1-3}$ Surgical stabilization of a flail chest injury in select patients can shorten the duration of ventilator support to reduce the morbidity and mortality associated with prolonged mechanical ventilation. ${ }^{4-9}$ Moreover, surgical stabilization can decrease longterm pain and disability of flail chest injury due to mal-unions, non-unions and progressive collapse of the flail segment. ${ }^{10-14}$ Recent guidelines published by the National Institute for Health

\footnotetext{
* Corresponding author. Tel.: +1 503413 5457; fax: +1 5034134942

E-mail address: mbottlan@lhs.org (M. Bottlang).
}

and Clinical Excellence (NICE) recommend surgical stabilization of a flail chest based on consistent evidence of its efficacy and lack of major safety concerns. ${ }^{15}$

Fixation of rib fractures with generic implants is challenging for several reasons: first, the cortex of ribs is on average less than $1 \mathrm{~mm}$ thick, ${ }^{16}$ providing little interface for reliable fixation of osteosynthesis implants, particularly in osteopenic bone. ${ }^{1}$ Second, ribs are highly flexible ${ }^{17,18}$ due to their ovoid cross-section with a typical width of $6-8 \mathrm{~mm} .{ }^{16}$ Standard osteosynthesis implants that do not restore the high flexibility of the native rib induce stress risers, are prone to fixation failure, and can lead to chest wall tightness. ${ }^{19,20}$ Third, the geometry of the rib surface is conical and twisted, making intraoperative contouring of generic plates timeconsuming and difficult. ${ }^{16,21}$

In case of flail chest injury, surgical stabilization of rib fractures is particularly difficult due to the number and complexity of fractures encountered and due to limited accessibility of posterior 
rib fractures. While not all fractures of a flail chest require fixation to stabilize a flail segment, the surgical procedure remains complex and typically requires a cooperative approach between orthopaedic, trauma or thoracic surgeons. ${ }^{2,22}$ This complexity may explain in part the finding of a recent survey among 238 surgeons, whereby $76 \%$ agreed that rib fracture fixation was indicated in select patients, but only $26 \%$ had ever performed or assisted in rib fracture repair. ${ }^{23}$ Surgical stabilization of flail chest injury has furthermore been complicated by a lack of implants that accommodate the unique form and function of ribs. ${ }^{19}$ For the past three decades, surgical stabilization of flail chest injury has primarily been performed with generic osteosynthesis plates and intramedullary Kirschner wires. $^{24}$ The majority of reported complications were implant-related, including screw pull-out, implant breakage, wire migration and cut-out, pain related to prone hardware and chest wall rigidity attributed to stiff implants. ${ }^{4,25-30}$

In order to reduce implant-related complications and to simplify the surgical technique, a dedicated implant system for rib fracture fixation (MatrixRIB, Synthes CMF, West Chester, PA) has recently been developed based on a biometric study of human ribs. ${ }^{16,17}$ This implant system entails anatomically contoured rib plates to reduce the need for intra-operative contouring, and intramedullary splints to enable less-invasive fixation of isolated or posterior fractures. ${ }^{17,21}$ Biomechanical studies demonstrated that anatomic plate constructs restored the stiffness and strength of native ribs ${ }^{17}$ and that intramedullary splint constructs were significantly stronger than Kirschner wire constructs while preventing cut-out failure. ${ }^{31,32}$ However, these bench-top studies can only predict clinical performance within the limited test parameters of the experimental setup.

This prospective observational study documented our clinical experience with MatrixRIB implants for stabilization of flail chest injury in 20 consecutive patients. Next to patient outcomes, this study assessed implant performance in terms of the amount of intra-operative implant contouring, the durability of implants, implant fixation and flail chest stabilization and the incidence of implant-related discomfort or chest tightness that would require implant removal.

\section{Patients and methods}

From January 2009 to January 2011, 20 consecutive patients that required surgical stabilization of flail chest injury were enrolled at the Level I Trauma Centres of (Legacy Health System in Portland, OR, and the University Medical Center Brackenridge, Austin, TX). Indications for surgical stabilization were flail chest injury with three or more consecutive ribs fractures in at least two locations. Exclusion criteria were age $<21$ years or $>80$ years, pregnancy, severe closed head injury, severe spinal cord injury and associated extra-thoracic injuries that made survival during the follow-up period unlikely. The study protocol was approved by the Internal Review Boards of both institutions.

Patient demographics and injury information was obtained from pertinent medical records. The number and location of rib fractures was extracted from three-dimensional computed tomography (CT) reconstructions (Fig. 1A). These pre-operative CT reconstructions were deemed essential to plan the surgical approach and stabilization. They were also used to quantitatively assess the amount of lung contusion. ${ }^{33}$ Areas corresponding to the healthy lung, contusion, haemothorax and pneumothorax were defined using a region segmentation tool (Amira 5.3, Visage Imaging GmbH, Berlin, Germany), and the contused volume was calculated as a percentage of the total long volume.

Flail chest stabilization was performed through a standard thoracotomy overlaying the flail location (Fig. 1B). Rib fractures were exposed and care was taken to preserve the periosteum (Fig. 1C). Fractures were stabilized with anatomic plates and intramedullary rib splints (Fig. 1D). For plate fixation, anatomically pre-contoured plates (MatrixRIB, Synthes) were cut to the desired

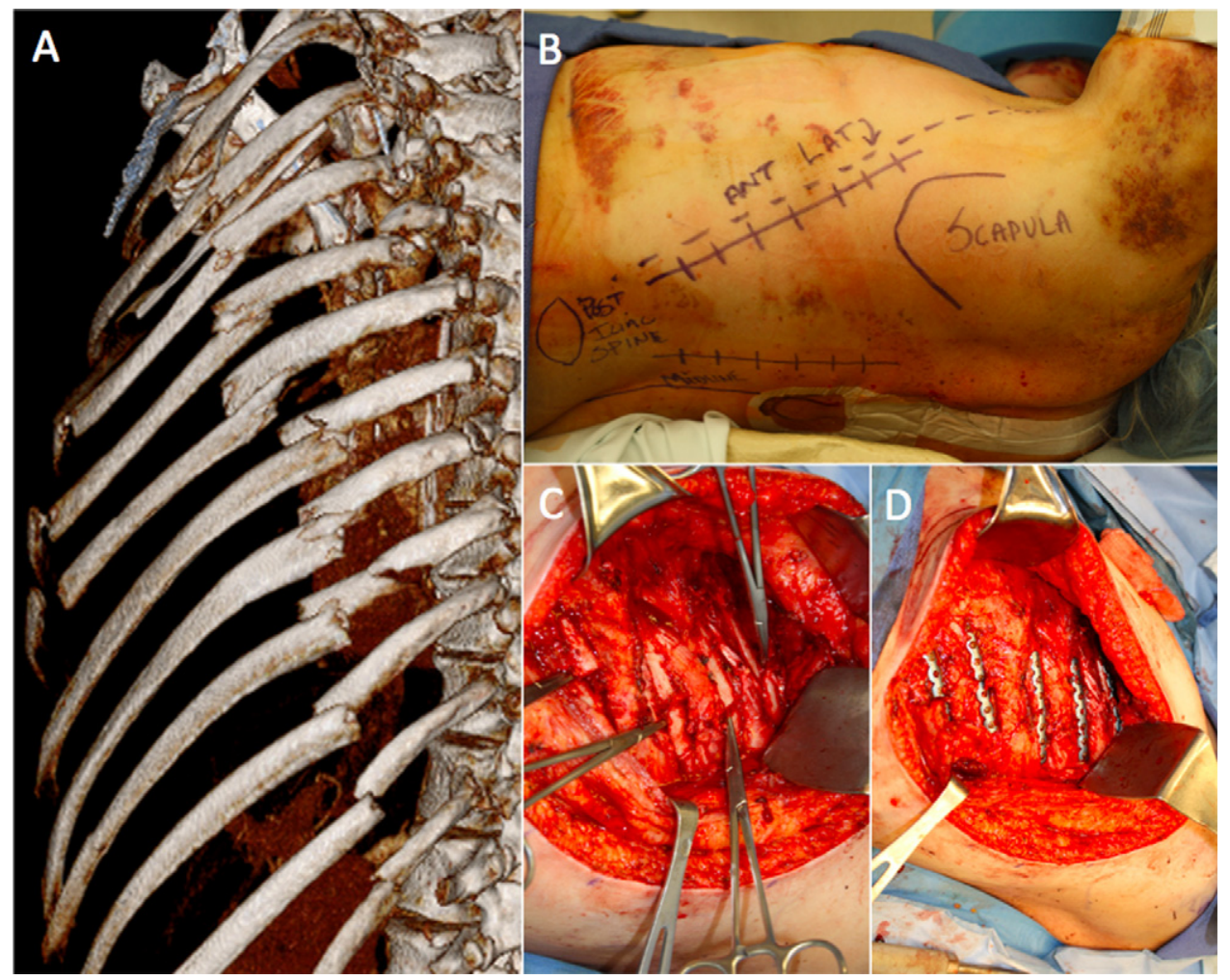

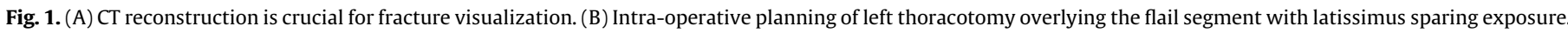
(C) Exposure of rib fracture with preservation of periosteum. (D) Surgical stabilization with anatomic plates. 


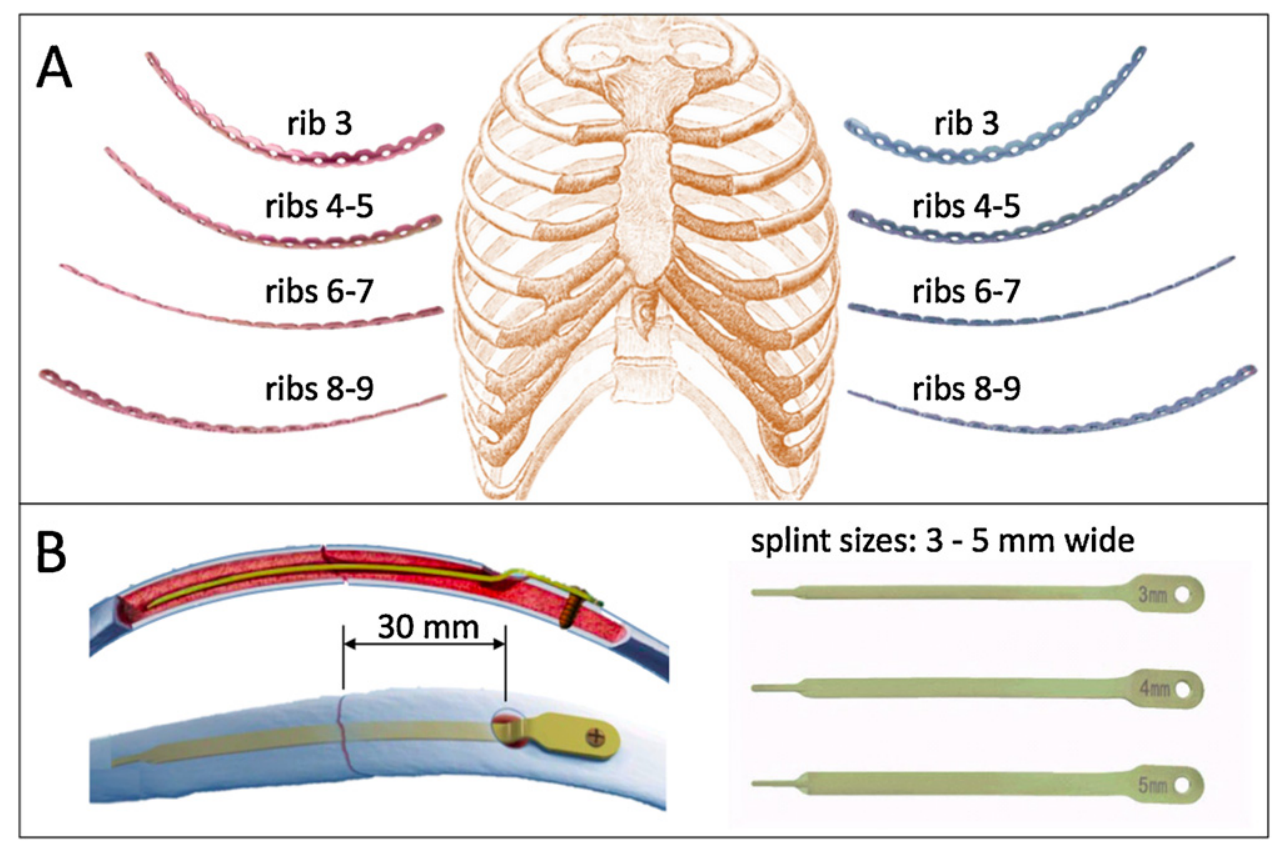

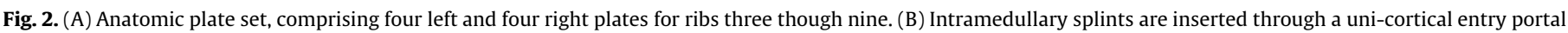
and advanced along the intramedullary canal across the fracture.

length (Fig. 2A). Plate fit was tested and corrected when necessary. The fractured rib segments were reduced to the plate and temporarily stabilized with plate holding forceps. A minimum of three self-tapping locking screws were inserted in each rib segment. When possible, a long plate was used to bridge both fractures of a flail rib segment. For intramedullary splinting, an entry portal was drilled $30 \mathrm{~mm}$ from the fracture (Fig. 2B). A rib splint (MatrixRIB, Synthes) was inserted into the entry portal, advanced across the fracture, and secured in the rib with a locking screw. The choice of plate versus splint fixation and the number of rib fractures requiring stabilization was left to the discretion of the operating surgeon. Upon flail chest stabilization, a chest tube was placed and a layered muscular closure with absorbable suture was performed.

The surgical procedure was documented by assessment of patient positioning, surgical approach, need for plate contouring, splint selection and intra-operative complications. Patient recovery was documented by assessment of post-operative pain management, duration of mechanical ventilation, ICU stay and hospitalization and by the incidence of pneumonia. Finally, patients were followed up at 3 and 6 months post surgery to assess pulmonary function, implant fixation and patient health. Pulmonary function was measured by spirometry and was expressed in terms of percentage forced vital capacity (\%FVC) and forced expiratory volume (\%FEV1), normalized to a healthy person of similar age, sex and body composition. Implant fixation was analysed on radiographs to assess implant or fixation failure. Patient health was evaluated with the RAND-36 general health survey encompassing eight components of health, ${ }^{34}$ and by the patients' ability to return to pre-injury activities.

\section{Results}

Of the 20 consecutive patients, one patient retracted study consent for reasons unrelated to implant performance. The remaining nineteen patients had a mean age of 50.7 years (range 29-70 years). Cause of injury included motor vehicle crash (8), fall from height (5), horse riding (2), crush (2), bicycle crash (1) and industrial blunt impact (1). Upon admission, patients had a mean Glasgow Coma Score of 13 (range 3-15), a mean Injury Severity Score of 28 (range 16-66) and a mean chest Abbreviated Injury Score (chest AIS) of 4.2 (range 4-5) (Table 1). Four patients (21\%) had at least one associated injury with AIS $\geq 4$. Contusion comprised on average $17 \%$ of the total long volume (range $0-$ $56 \%)$. Associated haemothorax and pneumothorax were visible on admission radiographs in 9 patients (47\%) and 14 patients (74\%), respectively.

Flail segments were antero-lateral in 6 patients (32\%), lateral in 4 patients (21\%) and postero-lateral in 9 patients (47\%). Patients had on average 8.5 fractured ribs (range $4-15$ ). The mean number of rib fractures per patient was 11.8 (range $5-21$ ), whereby $28 \%$ of fractures were anterior, $29 \%$ of fractures were lateral and $43 \%$ were posterior. Forty percent of fractures were comminuted.

Surgical stabilization was performed after a mean time of 5.3 days (range 1-17 days) from admission. On average, 6.2 fractures (range 3-9) were stabilized per patient, using on average 4.8 plates (range 2-9) and 0.8 splints (range 0-4) (Fig. 3). The total number of implants documented in this study included 91 plates and 15 splints, applied with 605 screws. The average plate length was 9.8 holes (range 5-17 holes). The average screw length was $10 \mathrm{~mm}$ (range $6-14 \mathrm{~mm}$ ). Contouring was required in 14 plates $(15 \%)$,

Table 1

Pre-operative injury assessment.

\begin{tabular}{|c|c|c|c|c|c|c|c|c|c|}
\hline \multirow[t]{2}{*}{ Category } & \multirow[t]{2}{*}{ GCS } & \multirow[t]{2}{*}{ ISS } & \multicolumn{6}{|l|}{ AIS } & \multirow{2}{*}{$\begin{array}{l}\text { Contusion } \\
\text { [\% volume] }\end{array}$} \\
\hline & & & Head, neck & Face & Chest & Abdomen & Extremity & External & \\
\hline Average & 13 & 28 & 1 & 0.3 & 4.2 & 1 & 1.4 & 0.5 & 17 \\
\hline Range & $3-15$ & $16-66$ & $0-5$ & $0-3$ & $4-5$ & $0-5$ & $0-4$ & $0-2$ & $0-56$ \\
\hline
\end{tabular}




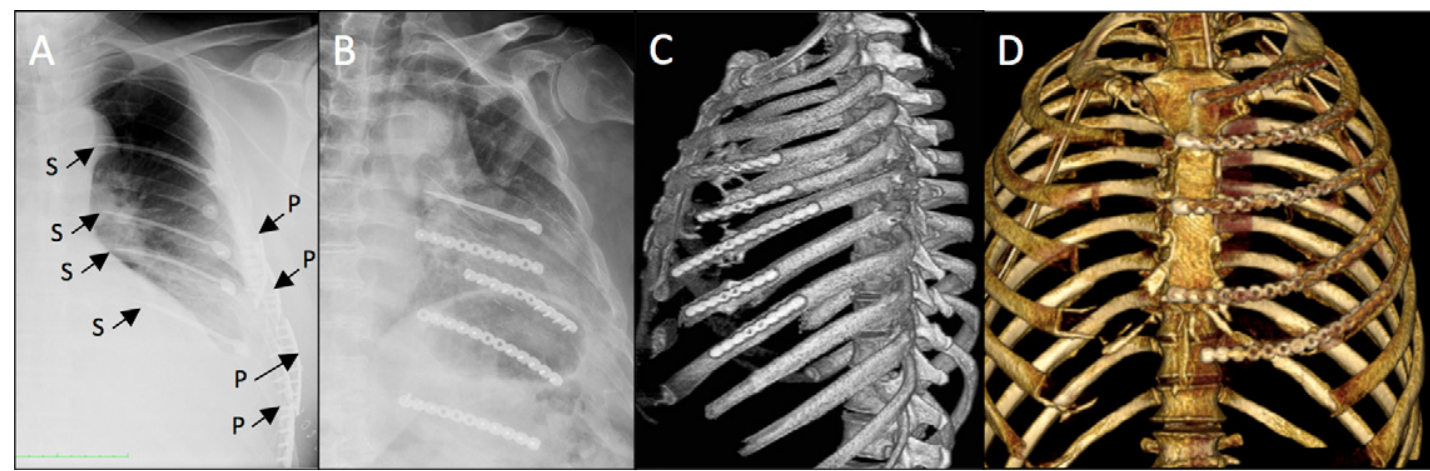

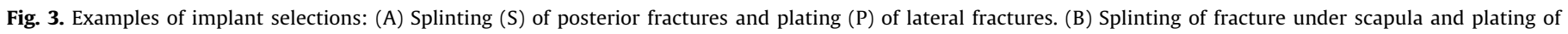
remaining fractures. (C) Plating of antero-lateral flail. (D) Spanning of costochondral junction with plates.

requiring less than 5 min per plate. The contoured plates were considerably long (mean length: 14.7 holes) and were used for bridging of the costochondral junction to obtain fixation in the sternum. Minor plate bending, requiring less than $1 \mathrm{~min}$ per plate, was occasionally performed to increase the plate curvature when plates were applied to the posterior rib segment. Post-operative radiographs demonstrated stable plate fixation until fracture union, with the exception of one posterior rib segment that was not appropriately reduced and fixed to the plate. This was asymptomatic and the fracture proceeded to heal uneventfully. Of the 15 splints, 13 splints (87\%) were used for posterior fractures and 2 splints were used for lateral fractures. Splint insertion encountered two minor complications. In one patient, the drill guide for the splint entry portal shifted off the centre of the rib during drilling, requiring drilling of a second entry portal. In another patient, the splint tip penetrated the outer cortex of a posterior rib segment during stabilization of a paravertebral rib fracture. The splint was partially retracted until its tip was visible at the fracture. After slightly bending the splint tip, splint insertion was completed uneventfully. Subsequently, bending of the splint tip at the fracture was used in three additional splints. Three surgeries were jointly performed by an orthopaedic surgeon and a thoracic surgeon. The remaining 16 surgeries were performed by either an orthopaedic surgeon (6), thoracic surgeon (7) or by a trauma surgeon (3). The duration of surgery as well as the time required for rib fracture reduction and stabilization are listed in Table 2, grouped by the number of implants being used per patient.

Post surgery, the mean duration of mechanical ventilation was 6.4 days (range $0-37$ days). Five patients (26\%) were weaned from ventilation within one day post surgery. One patient with

Table 2

Average duration of surgery, grouped by the number of implants being used per patient.

\begin{tabular}{lcc}
\hline Implants per patient & $\begin{array}{l}\text { Duration of surgery: } \\
\text { "cut-to-closure"a } \\
\text { [min] }\end{array}$ & $\begin{array}{l}\text { Duration of rib fracture } \\
\text { reduction + stabilization } \\
\text { [min] }\end{array}$ \\
\hline 3 & 125 & 50 \\
4 & 135 & 60 \\
5 & 167 & 90 \\
6 & 151 & 69 \\
7 & 234 & 101 \\
8 & 223 & 136 \\
9 & 230 & 120 \\
Average & $171 \pm 69$ & $83 \pm 39$ \\
\hline
\end{tabular}

a "Cut-to-closure" duration includes surgical approach, fracture reduction and stabilization, as well as time for additional procedures, such as thoracotomy, decortication, bronchoscopy, repair of lung and diaphragm lacerations, hernia repair and clavicle repair. associated injuries, an extremity AIS of 5, and a head and neck AIS of 4 require 37 days of ventilator support. Epidural analgesia was administered in 15 patients. The mean duration of epidural pain management post surgery was 6.6 days (range 2-18 days). The mean duration of Intensive Care Unit (ICU) stay and hospitalization was 7.9 days (range 1-34 days) and 18.4 days (range 4-68 days), respectively. Five patients (26\%) left the ICU within three days and were hospitalized for less than 10 days.

Postoperative complications were observed in 8 patients. These included pneumonia in 6 patients, atelectasis in two patients and wound infection in one patient. All patients with pneumonia and atelectasis recovered after treatment by fibreoptic bronchoscopy and antibiotics. One patient with a bilateral flail and sternal fracture developed a wound infection. During reoperation and wound debridement 30 days post the index surgery, the implants were removed and the chest wall was found to be stable. This was the only patient in which implants were removed.

Three and six months follow-up was obtained from 16 and 15 patients, respectively, with the remaining patients being lost to follow-up. At three months, patients regained an average \%FVC of $84 \%$ and a \%FEV1 of $77 \%$. At six month, the average \%FVC and \%FEV1 was $85 \%$ and $79 \%$, respectively. Return to pre-injury activity was achieved by five patients after 3 months and by seven patients after 6 months. These patients resumed pre-injury activities in construction (1), office work (4), management (1) and sales (1). Results of the 36 -item general health survey are summarized in Table 3 for each RAND category.

At six months follow-up, there was no implant breakage or hardware failure. All flail segments had healed in their stabilized position. None of the implants had migrated or lost their initial fixation. Discomfort related to implants was expressed by one of the 19 patients, reporting discomfort in the vicinity of two splints. No patient complained of chest tightness related to implants.

Table 3

RAND-36 general health survey (mean \pm standard deviation).

\begin{tabular}{lll}
\hline Category & 3 months & 6 months \\
\hline Physical function & $52 \pm 30$ & $54 \pm 31$ \\
Role limitation: physical & $16 \pm 31$ & $36 \pm 44$ \\
Role limitation: emotional & $64 \pm 38$ & $68 \pm 24$ \\
Social function & $53 \pm 32$ & $52 \pm 27$ \\
Mental health & $66 \pm 26$ & $65 \pm 24$ \\
Bodily pain & $39 \pm 35$ & $51 \pm 44$ \\
Vitality & $35 \pm 21$ & $58 \pm 26$ \\
General health & $66 \pm 18$ & $53 \pm 21$ \\
\hline
\end{tabular}




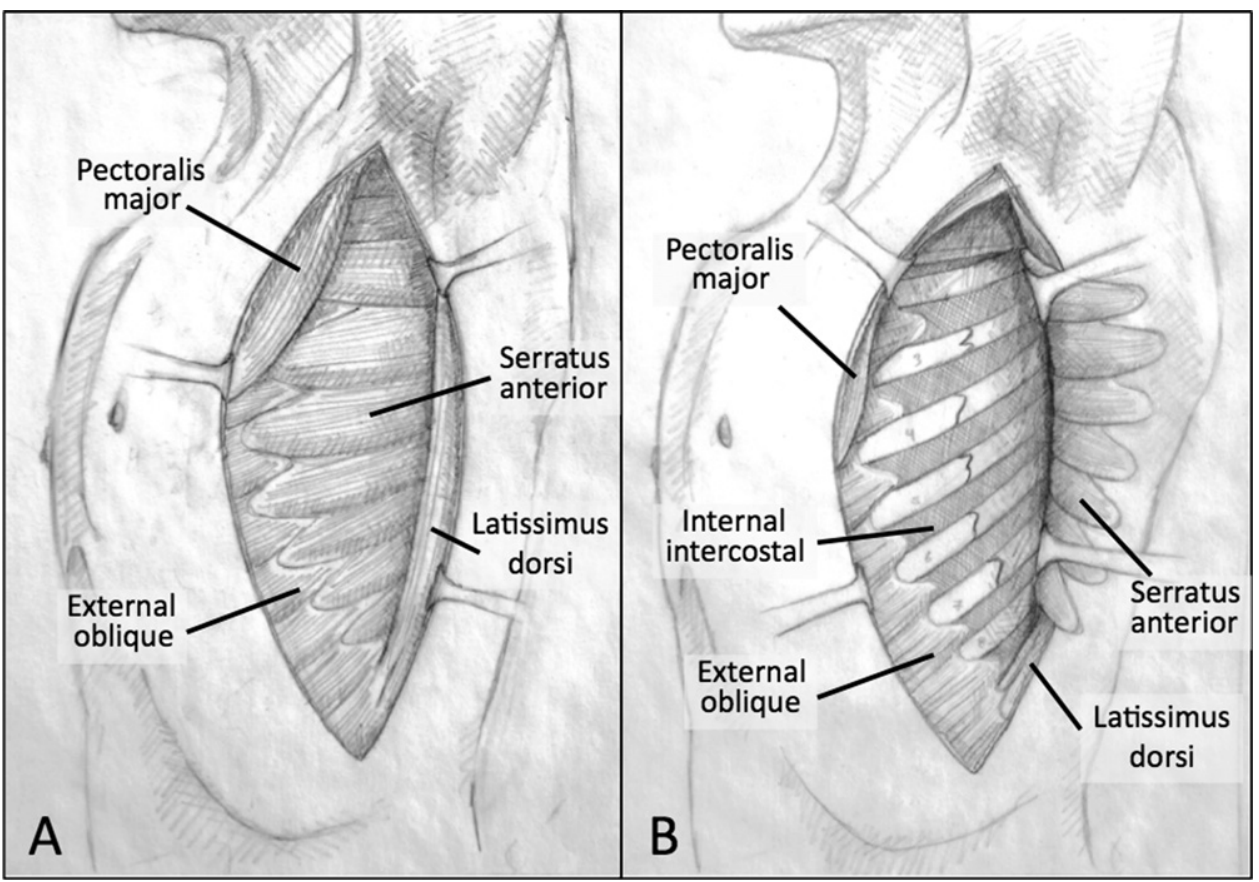

Fig. 4. Surgical approach: (A) Mobilization of the latissimus dorsi from its anterior border. (B) Access to lateral aspects of ribs 3-12.

\section{Discussion}

Results of this study demonstrate that anatomic rib plates provide reliable fixation and simplify the surgical procedure by eliminating the need for extensive intraoperative plate contouring. Contouring of anatomic plates was mostly limited to increasing the overall plate curvature when plates were applied to the posterior rib aspect, where ribs are more curved than at their anterior or lateral aspects. ${ }^{16}$ In cases of severe comminution, anatomic plates served as a valuable reduction tool. In all cases, rib plating restored chest wall stability. The use of long anatomic plates enabled bridging of both fractures adjacent to a flail rib segment with a single implant, which has been considered a "fundamental" benefit for stabilization of a flail chest. ${ }^{35}$ Plating a single rib of a flail chest segment yielded a marked stabilization of the flail segment. Therefore, it was deemed unnecessary to stabilize all fractured ribs. However, previous authors suggested that unstabilized fractures could develop non-unions and chronic pain, ${ }^{10,13,36}$ suggesting that all accessible rib fractures should be stabilized. Plate fixation with locking screws does not rely on compression of the plate onto the rib surface, but rather on the fixation of locking screws in the plate and rib. ${ }^{19}$ Interestingly, some locking screws were fixed in costochondral cartilage for plating of antero-lateral fractures and provided reliable fixation without fixation failure.

Intramedullary splints provided a less-invasive fixation alternative for single, non-comminuted fractures. However, the splint technique required a steeper learning curve than plating. Splints may not be used for severely comminuted fractures or for paraspinal fractures that do not provide sufficient intramedullary canal length to accommodate the splint.

The dedicated implant system facilitated the operative procedure to a level that allowed most cases to be performed by either an orthopaedic, thoracic or trauma surgeon. In contrast, prior studies emphasized the need for a team approach including orthopaedics to aid osteosynthesis with generic implants. ${ }^{2,22,37}$ Nevertheless, a team approach may still benefit the initial learning curve inherent to any new implant system. To facilitate fracture reduction, we recommend early stabilization of a flail chest, ideally, within one week of injury, to minimize the extent of dissection necessary for fracture mobilization.

The surgical exposure evolved over the course of this study towards minimizing muscle damage, facilitating wound closure, and preserving the serratus, latissimus, and skin as a connected functional unit. Ribs 3-12 may be exposed by mobilization of the latissimus dorsi from either its anterior border, posterior border or from both.

When mobilizing the anterior border of the latissimus, the serratus anterior is elevated from its anterior insertion points and kept as one unit with the latissimus (Fig. 4). The long thoracic and thoracodorsal nerves remain protected between the serratus and the latissimus. Alternatively, the serratus can be divided in line with its fibres after elevation of the latissimus, using one long vertical skin incision or a series of small horizontal incisions as described by Hasenboehler et al. ${ }^{36}$ Care must be taken when approaching the midaxillary line to preserve the long thoracic and thoracodorsal nerves.

When mobilizing the posterior border of the latissimus, a paraspinal incision about 4-6 cm off the midline was used (Fig. 5). The trapezius is mobilized towards the midline and may be released at its inferior border. With very superior, posterior fractures the rhomboid may also be elevated. The tendonis origin of the latissimus is detached posteriorly from the thoracolumbar fascia and mobilized anteriorly. The paraspinal muscles are exposed and mobilized from their lateral border towards the midline of the transverse processes, giving excellent exposure to posterior fractures of ribs 3-12.

The present study was limited to the clinical evaluation of MatrixRIB implants when used for surgical stabilization of flail chest injury. Patient outcomes correlated with those reported in previous papers on surgical stabilization of flail chest injury. ${ }^{4-}$ 6,8,9,38 Two prospective randomized studies reported a wound infection rate of $10-17 \%$ for surgical stabilization of flail chest, compared to $5 \%$ in the present study. ${ }^{6,9}$ They reported an incidence of pneumonia of $12-22 \%$, compared to $26 \%$ in the present study. For patients without surgical stabilization, they reported 12-18 days post-operative ventilation and 15-27 days ICU stay, compared to 


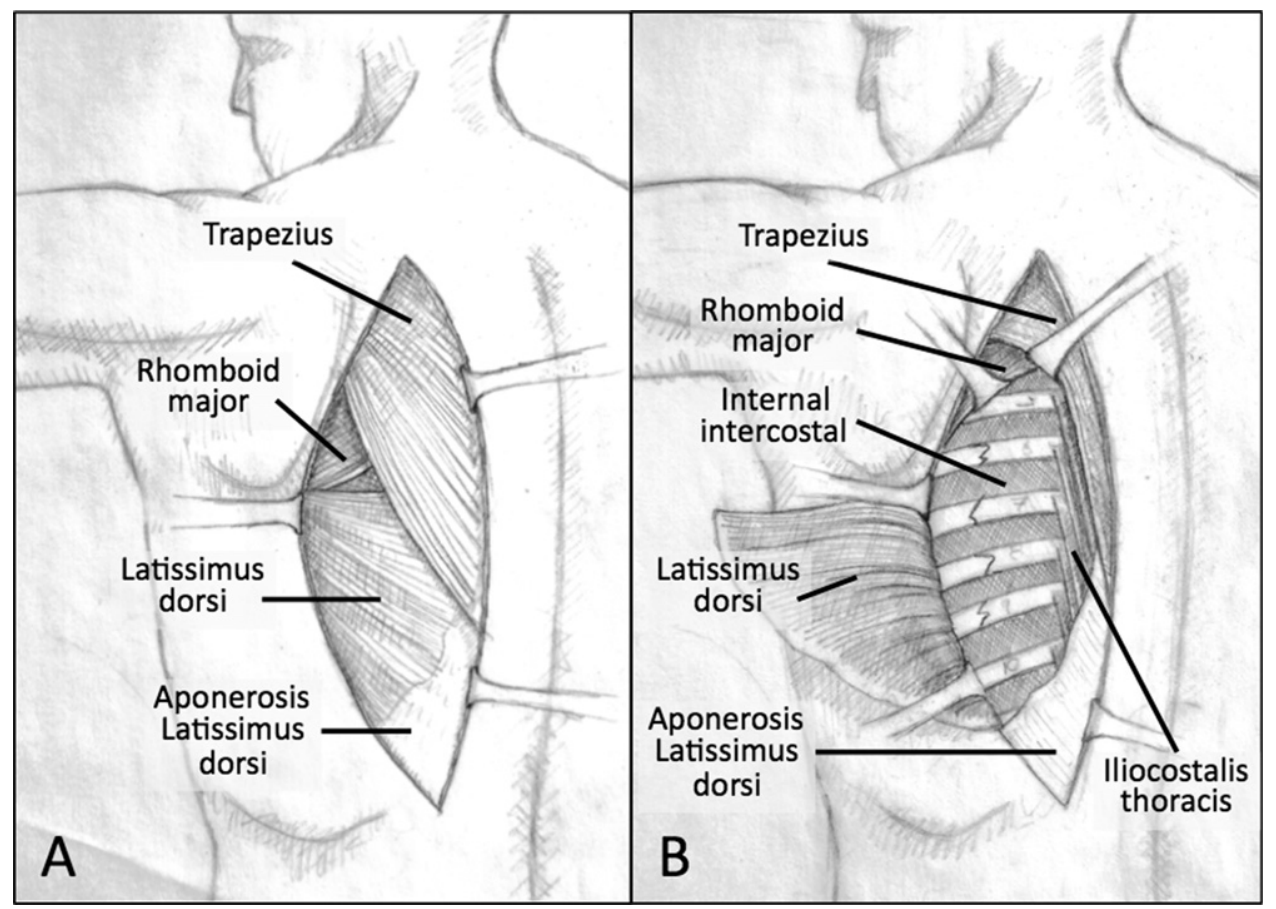

Fig. 5. Surgical approach: (A) Mobilization of the latissimus dorsi through a paraspinal incision. (B) Access to posterior rib fractures.

6.4 days ventilation and 7.9 days ICU stay after surgical stabilization in the present study. At six months post surgical stabilization, Tanaka et al. reported lung capacity (\%FVC) of $95 \%$, whereby $61 \%$ of patients had returned to full-time employment. ${ }^{9}$ In the present study, \%FVC was $86 \%$ and $47 \%$ of patients returned to pre-injury activities at 6 months. In stark contrast, $50-60 \%$ of patients with flail chest injury treated non-operatively develop disabling long-term morbidities. ${ }^{12,39}$ One long-term study reported that $39 \%$ of flail chest patients did not returned to work at 5 years post injury. ${ }^{39}$ Studies by Ahmed and Mohyuddin and Granetzny et al. reported mortality rates of $8-10 \%$ for surgical stabilization and $15-29 \%$ for non-surgical treatment of flail chest. $^{4,6}$ There was no mortality in the present study. They utilized Kirschner wires and wire cerclage and observed loss of stabilization of the flail segment in $5-13 \%$. There was no loss of flail stabilization in the present study. Finally, while this study did not analyse the treatment cost, two prior studies demonstrated that surgical stabilization decreased hospital costs by up to $43 \%$ when compared to a non-operative control group. ${ }^{5,9}$ The potential socioeconomic benefit of surgical stabilization is furthermore supported by the dramatic increase in return to full-time employment compared to non-surgical stabilization. ${ }^{9}$

In conclusion, results of this study demonstrate that anatomic plates largely eliminated the need for plate contouring. Long plates enabled bridging of a flail segment with a single implant. Splints provided an intramedullary fixation alternative for simple fractures and did not exhibit implant migration and cut-out seen with traditional Kirschner wires. There was no mortality, no implant breakage, no failure of initial implant fixation, no nonunions and all flail segments remained stable. Therefore, results of this study support the clinical use of anatomic plates and splints for stabilization of flail chest injury.

\section{Conflict of interest statement}

The authors Michael Bottlang, William B. Long, and Steven Madey receive royalties and consulting income from Synthes CMF related to MatrixRIB implants.

\section{Acknowledgements}

Financial support for the conduct of this study has been provided by Synthes CMF, West Chester, PA, USA. The study sponsor had no involvement in the study design, data analysis, manuscript preparation or decision on publication.

\section{References}

1. Lafferty PM, Anavian J, Will RE, Cole PA. Operative treatment of chest wall injuries: indications, technique, and outcomes. Journal of Bone and Joint Surgery 2011;93(1):97-110.

2. Nirula R, Mayberry JC. Rib fracture fixation: controversies and technical challenges. American Surgeon 2010;76(8):793-802.

3. Wanek S, Mayberry JC. Blunt thoracic trauma: flail chest, pulmonary contusion, and blast injury. Critical Care Clinics 2004;20(1):71-81.

4. Ahmed Z, Mohyuddin Z. Management of flail chest injury: internal fixation versus endotracheal intubation and ventilation. Journal of Thoracic and Cardiovascular Surgery 1995;110(6):1676-80.

5. Althausen PL, Coll D, O'Mara T, Bray TJ. Surgical stabilization of flail chest with locked plate fixation. Proceedings of the 26th annual meeting of orthopaedic trauma association, vol. 67. 2010:210.

6. Granetzny A, Abd El-Aal M, Emam E, Shalaby A, Boseila A. Surgical versus conservative treatment of flail chest. Evaluation of the pulmonary status. Interactive Cardiovascular and Thoracic Surgery 2005;4(6):583-7.

7. Karev DV. Operative management of the flail chest. Wiadomosci Lekarskie 1997;50(Suppl. 1 Pt 2):205-8.

8. Nirula R, Allen B, Layman R, Falimirski ME, Somberg LB. Rib fracture stabilization in patients sustaining blunt chest injury. American Surgeon 2006;72(4):307-9.

9. Tanaka H, Yukioka T, Yamaguti Y, Shimizu S, Goto H, Matsuda H, et al. Surgical stabilization of internal pneumatic stabilization? A prospective randomized study of management of severe flail chest patients. Journal of Trauma 2002;52(4):727-32. [discussion 32].

10. Cacchione RN, Richardson JD, Seligson D. Painful nonunion of multiple rib fractures managed by operative stabilization. Journal of Trauma 2000;48(2): 319-21.

11. Kerr-Valentic MA, Arthur M, Mullins RJ, Pearson TE, Mayberry JC. Rib fracture pain and disability: can we do better? Journal of Trauma 2003;54(6):1058-63. [discussion 63-4].

12. Mayberry JC, Kroeker AD, Ham LB, Mullins RJ, Trunkey DD. Long-term morbidity, pain, and disability after repair of severe chest wall injuries. American Surgeon 2009;75(5):389-94.

13. Ng AB, Giannoudis PV, Bismil Q, Hinsche AF, Smith RM. Operative stabilisation of painful non-united multiple rib fractures. Injury 2001;32(8):637-9.

14. Richardson JD, Franklin GA, Heffley S, Seligson D. Operative fixation of chest wall fractures: an underused procedure? American Surgeon 2007;73(6):591-6. [discussion 6-7]. 
15. NICE: National Institute for Health and Clinical Excellence. Insertion of metal rib reinforcements to stabilise a flail chest wall. 2010 IPG361 http://egap.evidence.nhs.uk/ IPG361.

16. Mohr M, Abrams E, Engel C, Long WB, Bottlang M. Geometry of human ribs pertinent to orthopedic chest-wall reconstruction. Journal of Biomechanics 2007;40(6):1310-7.

17. Bottlang M, Walleser S, Noll M, Honold S, Madey SM, Fitzpatrick D, et al Biomechanical rationale and evaluation of an implant system for rib fracture fixation. European Journal of Trauma and Emergency Surgery 2010;36(5):417-26.

18. Schultz AB, Benson DR, Hirsch C. Force-deformation properties of human ribs. Journal of Biomechanics 1974;7(3):303-9.

19. Engel C, Krieg JC, Madey SM, Long WB, Bottlang M. Operative chest wall fixation with osteosynthesis plates. Journal of Trauma 2005;58(1):181-6.

20. Labitzke R. From "bone suture" to modern osteosynthesis-a chronology Chirurg 1995;66(4):452-8.

21. Bottlang M, Helzel I, Long WB, Madey S. Anatomically contoured plates for fixation of rib fractures. Journal of Trauma 2010;68(3):611-5.

22. Bemelman M, Poeze M, Blokhuis TJ, Leenen LP. Historic overview of treatment techniques for rib fractures and flail chest. European Journal of Trauma and Emergency Surgery 2010;36(5):407-15.

23. Mayberry JC, Ham LB, Schipper PH, Ellis TJ, Mullins RJ. Surveyed opinion of American trauma, orthopedic, and thoracic surgeons on rib and sternal fracture repair. Journal of Trauma 2009;66(3):875-9.

24. Fitzpatrick DC, Denard PJ, Phelan D, Long WB, Madey SM, Bottlang M. Operative stabilization of flail chest injuries: review of literature and fixation options. European Journal of Trauma and Emergency Surgery 2010;36(5):427-33.

25. Hellberg K, de Vivie ER, Fuchs K, Heisig B, Ruschewski W, Luhr HG, et al. Stabilization of flail chest by compression osteosynthesis-experimental and clinical results. Thoracic and Cardiovascular Surgeon 1981;29(5):275-81.

26. Lardinois D, Krueger T, Dusmet M, Ghisletta N, Gugger M, Ris HB. Pulmonary function testing after operative stabilisation of the chest wall for flail chest European Journal of Cardio-Thoracic Surgery 2001;20(3):496-501.

27. Moore BP. Operative stabilization of nonpenetrating chest injuries. Journal of Thoracic and Cardiovascular Surgery 1975;70(4):619-30.
28. Mouton W, Lardinois D, Furrer M, Regli B, Ris HB. Long-term follow-up of patients with operative stabilisation of a flail chest. Thoracic and Cardiovascular Surgeon 1997; $45(5): 242-4$.

29. Shah TJ. On internal fixation for flail chest. Journal of Thoracic and Cardiovascular Surgery 1996;112(3):849-50.

30. Voggenreiter G, Neudeck F, Aufmkolk M, Obertacke U, Schmit-Neuerburg KP. Operative chest wall stabilization in flail chest-outcomes of patients with or without pulmonary contusion. Journal of the American College of Surgeons 1998;187(2):130-8.

31. Bottlang M, Helzel I, Long W, Fitzpatrick D, Madey S. Less-invasive stabilization of rib fractures by intramedullary fixation: a biomechanical evaluation. Journal of Trauma 2010;68(5):1218-24.

32. Helzel I, Long W, Fitzpatrick D, Madey S, Bottlang M. Evaluation of intramedullary rib splints for less-invasive stabilisation of rib fractures. Injury 2009;40(10):1104-10.

33. Daly M, Miller PR, Carr JJ, Gayzik FS, Hoth JJ, Meredith JW, et al. Traumatic pulmonary pathology measured with computed tomography and a semiautomated analytic method. Clinical Imaging 2008;32(5):346-54.

34. Hays RD, Morales LS. The RAND-36 measure of health-related quality of life. Annals of Medicine 2001;33(5):350-7.

35. Sanchez-Lloret J, Letang E, Mateu M, Callejas MA, Catalan M, Canalis E, et al. Indications and surgical treatment of the traumatic flail chest syndrome. An original technique. Thoracic and Cardiovascular Surgeon 1982;30(5):294-7.

36. Hasenboehler EA, Bernard AC, Bottiggi AJ, Moghadamian ES, Wright RD, Chang PK, et al. Treatment of traumatic flail chest with muscular sparing open reduction and internal fixation: description of a surgical technique. Journal of Trauma 2011;71(2):494-501.

37. Althausen PL, Shannon S, Watts C, Thomas K, Bain MA, Coll D, et al. Early surgical stabilization of flail chest with locked plate fixation. Journal of Orthopaedic Trauma 2011;25:641-8.

38. Balci AE, Eren S, Cakir O, Eren MN. Open fixation in flail chest: review of 64 patients. Asian Cardiovascular and Thoracic Annals 2004;12(1):11-5.

39. Landercasper J, Cogbill TH, Lindesmith LA. Long-term disability after flail chest injury. Journal of Trauma 1984;24(5):410-4. 